\title{
Spin structure factors and valence-bond-solid states of the trimerized Heisenberg chains in a magnetic field
}

\author{
Shou-Shu Gong, Bo Gu and Gang Su* \\ College of Physical Sciences, Graduate University of Chinese Academy of Sciences, \\ P. O. Box 4588, Beijing 100049, China
}

\begin{abstract}
By means of the density matrix renormalization group (DMRG) method, the static spin structure factors and the magnetization plateaus of the trimerized Heisenberg ferromagnet-ferromagnetantiferromagnet and antiferromagnet-antiferromagnet-ferromagnet spin chains in the presence of a magnetic field are elaborately studied. It is found that in the plateau states, the static structure factor with three peaks does not vary with the external magnetic field as well as the exchange couplings; the spin correlation function behaves as a perfect sequence and has a simple relation with the magnetization per site. An approximate wave function for the plateau states is proposed, and a picture based on the valence-bond-solid states is presented in order to understand the origin and the total number of the magnetization plateaus, which are shown to be in agreement with the DMRG results.
\end{abstract}

PACS numbers: 75.10.Jm, 75.40.Cx 
In recent years, owing to their unusual magnetic properties, the low-dimensional quantum spin systems have been extensively investigated both theoretically and experimentally (see e.g. Ref. [1]). Among others, the phenomenon of topological quantization of magnetization has received particular attention (e.g. [2, 3, 4, 15, 6, 7, 8, 9, 10, 11, 12, 13, 14, 15, 16, 17]). Recently, the magnetic and thermodynamic properties of the ferromagnet-ferromagnetantiferromagnet (F-F-AF) and antiferromagnet-antiferromagnet-ferromagnet (AF-AF-F) trimerized Heisenberg spin chains have been systematically explored[14, 15]. For both systems, it have been observed that the topological quantization condition $n(S-m)=$ integer, with $n$ the period of the ground state determined by the explicit spatial structure of Hamiltonian, $S$ the spin, and $m$ the magnetization per site, is only a necessary, but not a sufficient, condition for the appearance of magnetization plateaus, and the total number of the plateaus is $2 S+1$. The width of plateaus is found to decrease with increasing the ferromagnetic interaction, and the plateau-non-plateau transition is of Kosterlitz-Thouless type, while the position of the plateaus is independent of the magnitude of the exchange couplings [14]. The magnetization plateau is smeared out when temperature is increased, and it appears the systems fall into different thermodynamic states under different magnetic fields[15]. In order for understanding the spin-spin correlations in such intriguing systems, and demonstrating why the total number of the plateaus is $2 S+1$, we shall, in this paper, investigate the static spin structure factor that would be directly measured experimentally for F-F-AF and AF-AF-F trimerized Heisenberg chains with $S=1 / 2$ and 1 , and offer a picture based on the valence-bond-solid (VBS) states for further understanding the origin and the total number of the plateaus.

The Hamiltonian of the trimerized Heisenberg spin chain in a magnetic field is given by

$$
\begin{aligned}
H & =\sum_{j}\left(J \mathbf{S}_{3 j-2} \cdot \mathbf{S}_{3 j-1}+J \mathbf{S}_{3 j-1} \cdot \mathbf{S}_{3 j}\right. \\
& \left.+J^{\prime} \mathbf{S}_{3 j} \cdot \mathbf{S}_{3 j+1}\right)-h \sum_{j} S_{j}^{z},
\end{aligned}
$$

where $J, J^{\prime}>0$ denote the AF coupling and $<0$ the $\mathrm{F}$ coupling, and $h$ is the external magnetic field. We take $g \mu_{B}=1$ for simplicity. Two cases with $S=1 / 2$ and 1 will be considered: (a) $J=-J_{F}<0, J^{\prime}=J_{A F}>0$, the system (F-F-AF) is an antiferromagnet; (b) $J=J_{A F}>0, J^{\prime}=-J_{F}<0$, the system (AF-AF-F) is a ferrimagnet. The static structure 
factor $S(q)$ is the Fourier transform of the spin correlation function $\left\langle S_{n}^{z} S_{n^{\prime}}^{z}\right\rangle$, defined by [18]

$$
S(q)=\frac{1}{N} \sum_{n, n^{\prime}} e^{i q\left(n-n^{\prime}\right)}\left\langle S_{n}^{z} S_{n^{\prime}}^{z}\right\rangle .
$$

The density matrix renormalization group (DMRG) method [19, 20] will be invoked. In the following calculations, the length of the chain is taken as $N=60$, and the number of the optimal states is kept as $M=60$. Open boundary conditions are adopted. The truncation error is less than $10^{-3}$ in all calculations.

For the spin-1/2 F-F-AF chain with couplings $J_{A F} / J_{F}=1$, one magnetization plateau with $m=1 / 6$ is observed, being consistent with the quantization condition [5]. Let $h_{c 1}$ and $h_{c 2}$ be the lower and upper critical fields at which the magnetization plateau appears and disappears, respectively, and $h_{s}$ be the saturation field, as indicated in Fig. 11(a). When $h<h_{c 1}$, as demonstrated in Fig. 1(b), the static spin structure factor $S(q)$ shows three peaks at $q=\pi / 3,5 \pi / 3$, and $\pi$. With increasing the magnetic field, the peak at $q=\pi$ disappears while the peak at $q=\pi / 3(5 \pi / 3)$ splits into two small peaks that continuously shift towards $q=0$ and $2 \pi / 3(q=0$ and $4 \pi / 3)$, respectively. Meanwhile, new peaks start to appear in $q=0,2 \pi / 3$ and $4 \pi / 3$. When $h_{c 1}<h<h_{c 2}$, where the ground states are in the plateau state with $m=1 / 6$, as shown in Fig. 1(c), $S(q)$ with three peaks at $q=0,2 \pi / 3$ and $4 \pi / 3$ does not vary with the magnetic field, which corresponds to the long-range order and a perfect sequence for the spin correlation function $\left\langle S_{0}^{z} S_{j}^{z}\right\rangle$, which we will discuss later. When $h_{c 2}<h<h_{s}$, the peaks at $q=2 \pi / 3$ and $4 \pi / 3$ are suppressed with the increasing field and disappear at the saturation field, while the peak at $q=0$ increases with the field, which show the ferromagnetic characteristic, as demonstrated in Fig. 1(d).

In the absence of the external field, we find that the static structure factor $S(q)$ could be fitted by

$$
\begin{aligned}
S(q) & =\sum_{l=1}^{3}\left\{\alpha _ { l } e ^ { - l \beta } \left[\frac{2 \sin 3 q(\cosh 3 \beta-\cos 3 q) \sin l q}{\cosh (6 \beta)-\cos (6 q)}\right.\right. \\
& \left.\left.+\frac{\left(e^{6 \beta}-\cos 6 q-2 \cos 3 q \sinh 3 \beta\right) \cos l q}{\cosh (6 \beta)-\cos (6 q)}\right]\right\} \\
& +\frac{S(S+1)}{3}
\end{aligned}
$$

where $\alpha_{l}$ and $\beta$ are parameters. As presented in Fig. 1(b), the characteristic peaks can be well fitted by Eq. (3), with only a slightly quantitative derivation, showing that the main 
features of $S(q)$ of this model can be captured by a linear superposition of three modes, which is closely related to the low-lying excitations of these trimerized spin systems [14, 17].

In the plateau states, $S(q)$ has three peaks at $q=0,2 \pi / 3$ and $4 \pi / 3$, and $\left\langle S_{0}^{z} S_{j}^{z}\right\rangle$ shows a perfect sequence with a period of 3 . It is the period of $\left\langle S_{0}^{z} S_{j}^{z}\right\rangle$ that determines the peak positions of $S(q)$. Define $\xi(m)=(1 / N) \sum_{n}\left\langle S_{n}^{z} S_{n-m}^{z}\right\rangle$, which also shows a perfect sequence with a period of 3 , say, $\{\cdots,(\xi(3 j), \xi(3 j+1), \xi(3 j+2)), \cdots\}$. Thus, the structure factor $S(q)$ in the plateau states can be written as

$$
\begin{aligned}
S(q) & =\frac{1}{3} S(S+1)+2 \xi(1) \cos (q)+2 \xi(2) \cos (2 q) \\
& +2 \sum_{j=1}^{\infty} \xi(3 j) \cos (3 j q)+2 \sum_{j=1}^{\infty} \xi(3 j+1) \cos (3 j+1) q \\
& +2 \sum_{j=1}^{\infty} \xi(3 j+2) \cos (3 j+2) q .
\end{aligned}
$$

Because the series $\sum_{j=1}^{\infty} \cos (3 j q)$ only diverges at $q=0,2 \pi / 3$ and $4 \pi / 3$, and oscillates at other $q$, the peak positions of $S(q)$ appear only at $q=0,2 \pi / 3$ and $4 \pi / 3$, consistent with our numerical calculations. This argumentation may be generalized to other spin chains, say, if $\left\langle S_{0}^{z} S_{j}^{z}\right\rangle$ behaves as a perfect sequence with a period of $n, S(q)$ would only have peaks at $q=0,2 \pi / n, 4 \pi / n, \cdots$.

Besides the sequence structure of the spin correlation function, it is found that, from our DMRG results, in the plateau states the spin correlation functions satisfy the following relation:

$$
\frac{1}{3}[\xi(3 j)+\xi(3 j+1)+\xi(3 j+2)]=m^{2},
$$

which does not alter with the couplings $J_{A F} / J_{F}$ as well as the magnitude of the spin $S$. As the susceptibility $\chi(T)=\frac{1}{k_{B} T} \frac{1}{N} \sum_{i, j}\left[\left\langle S_{i}^{z} S_{j}^{z}\right\rangle-\left\langle S_{i}^{z}\right\rangle\left\langle S_{j}^{z}\right\rangle\right]$ bears a finite value at $T \rightarrow 0$ in the plateau states because of $m(T)$ being a constant, it leads to

$$
\frac{1}{N} \sum_{i, j}\left[\left\langle S_{i}^{z} S_{j}^{z}\right\rangle-\left\langle S_{i}^{z}\right\rangle\left\langle S_{j}^{z}\right\rangle\right]=0
$$

at $T \rightarrow 0$. Since $\left\langle S_{0}^{z} S_{j}^{z}\right\rangle$ shows a perfect sequence in the plateau states, we can get Eq. (5) directly from Eq. ([6]).

The spin-1 F-F-AF Heisenberg chain with the couplings $J_{A F} / J_{F}=1$ is also studied. The two plateaus at $m=1 / 3$ and $2 / 3$ are observed. The static structure factor and spin correlation function show the same qualitative characteristics as those of the spin- $1 / 2$ chain. 

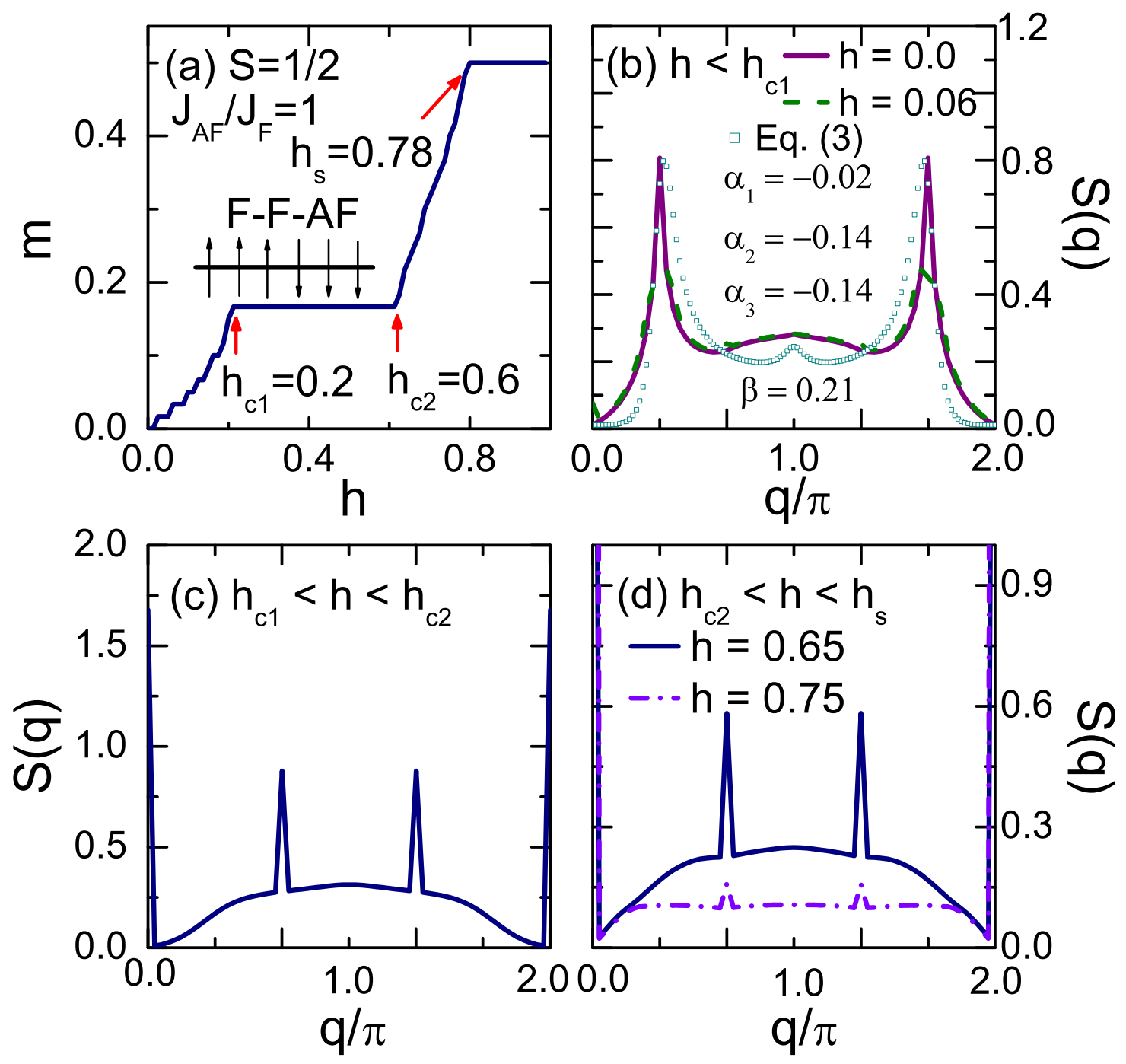

FIG. 1: (Color online) For a spin-1/2 F-F-AF trimerized Heisenberg chain with couplings $J_{A F} / J_{F}=$ 1, (a) the magnetization per site $m$ as a function of the external magnetic field $h$ in the ground states; and the static structure factor $S(q)$ in the ground states under the external field (b) $h<h_{c 1}$, (c) $h_{c 1}<h<h_{c 2}$, and (d) $h_{c 2}<h<h_{s}$.

The zero-field static structure factor $S(q)$ can also be well fitted by Eq. (3), and in both plateaus, Eq. (5) is still satisfied.

The AF-AF-F trimerized Heisenberg chains with spin $1 / 2$ and 1 are investigated as well. For the spin-1 AF-AF-F Heisenberg chain with couplings $J_{A F} / J_{F}=1$, two plateaus at 
$m=1 / 3$ and $m=2 / 3$ are observed. Let $h_{c 1}$ denote the critical field of disappearance of $m=1 / 3$ plateau, $h_{c 2}$ and $h_{c 3}$ be the critical fields of appearance and disappearance of $m=2 / 3$ plateau, respectively, and $h_{s}$ be the saturation field, as marked in Fig. 2(a). When no magnetic field is applied, $S(q)$ shows three peaks at $q=0,2 \pi / 3$ and $4 \pi / 3$, which differs from the case of the F-F-AF chain because the latter is an antiferromagnet. When $h<h_{c 1}$ and $h_{c 2}<h<h_{c 3}$, where the ground states are in $m=1 / 3$ and $2 / 3$ plateau states, $S(q)$ with three peaks at $q=0,2 \pi / 3$ and $4 \pi / 3$ does not vary with the external field, both of which show a perfect sequence with a period of 3 for $\left\langle S_{0}^{z} S_{j}^{z}\right\rangle$, as shown in Fig. 2(b). When $h_{c 3}<h<h_{s}$, like the case of the F-F-AF chain, the peaks at $q=2 \pi / 3$ and $4 \pi / 3$ are depressed with increasing the field, and disappear at the saturation field, while the peak at $q=0$ increases with the field, as shown in Fig. 2(d).

The spin-1/2 AF-AF-F Heisenberg chain with couplings $J_{A F} / J_{F}=1$ is also explored. The features similar to those of $S=1$ are seen, and the spin correlation functions also satisfy Eq. (5) in the plateau states.

The plateau states for the F-F-AF and AF-AF-F trimerized Heisenberg chains with spin $S$ can be described by an approximate wave function defined by

$$
\begin{aligned}
\psi_{i} & =a\left|\uparrow_{3 i-2} \uparrow_{3 i-1} \downarrow_{3 i}\right\rangle+b\left|\uparrow_{3 i-2} \downarrow_{3 i-1} \uparrow_{3 i}\right\rangle \\
& +c\left|\downarrow_{3 i-2} \uparrow_{3 i-1} \uparrow_{3 i}\right\rangle, \quad(i=1, \cdots, N / 3)
\end{aligned}
$$

where $\uparrow_{j}\left(\downarrow_{j}\right)$ denotes spin up (down) on site $j$. It is found that, if the coefficients $a, b$ and $c$ are properly chosen, one could find the so-calculated local magnetic moment $\left\langle S_{j}^{z}\right\rangle$ as well as the spin correlation functions to be in agreement with the DMRG results. Take the spin1/2 F-F-AF trimerized Heisenberg chain for example. In the plateau state with $m=1 / 6$, the DMRG results show that $\left\langle S_{j}^{z}\right\rangle$ behaves as $\left\{\cdots,\left(S_{1}, S_{2}, S_{2}\right), \cdots\right\}$ with $S_{1}=0.367$ and $S_{2}=0.067$, giving rise to the magnetization per site $m=\left(S_{1}+2 S_{2}\right) / 3=1 / 6$, and the spin correlation function $\xi(j)$ behaves such that $\{\cdots,(\xi(3 j), \xi(3 j+1), \xi(3 j+2)), \cdots\}$ with $\xi(3 j)=$ 0.048 and $\xi(3 j+1)=\xi(3 j+2)=0.018$, satisfying $\frac{1}{3}[\xi(3 j)+\xi(3 j+1)+\xi(3 j+2)]=\left(\frac{1}{6}\right)^{2}$. If we choose the coefficients $a= \pm \sqrt{13 / 30}, b= \pm \sqrt{13 / 30}$ and $c= \pm \sqrt{2 / 15}$, from the approximate wave function we can obtain $\left\langle\psi_{i}\left|S_{3 i-2}^{z}\right| \psi_{i}\right\rangle=11 / 30,\left\langle\psi_{i}\left|S_{3 i-1}^{z}\right| \psi_{i}\right\rangle=\left\langle\psi_{i}\left|S_{3 i}^{z}\right| \psi_{i}\right\rangle=1 / 15$, and $\xi(3 j)=43 / 900, \xi(3 j+1)=\xi(3 j+2)=4 / 225$, both of which are quite in agreement with our DMRG results.

Let us now invoke the VBS picture to show why the total number of the magnetization 
plateaus in these trimerized systems is $2 S+1$. As shown in Fig. [3, we denote a spin $1 / 2$ by a bullet, a spin singlet of two spin $1 / 2$ by a short line between two bullets, and the spin $S$ per site by a large open ellipse that could be viewed as a combination of $2 S$ spin $1 / 2$. For the F-F-AF chains with spin $S=1 / 2,1,3 / 2$ and 2 , the isolated spins $S$ that are denoted by large open ellipses, are separated by spin singlet states. Thus, these isolated spins could be regarded as a classic spin chain with antiferromagnetic couplings, whose magnetization curves should have no plateau except for the saturation plateau of $m=S / 3$. This picture explains why the magnetization plateau of $m<S / 3$, which is permitted by the quantization condition [5], does not appear. For the ferrimagnetic AF-AF-F chains with $S=1 / 2,1,3 / 2$ and 2 , the isolated spins are aligned parallel, and the magnetization per site in the absence of the magnetic field is $S / 3$, leading to the plateaus of $m<S / 3$ never exist. With increasing the magnetic field, the spin singlet bonds are gradually broken. When a spin singlet bond per three sites is simultaneously broken, a new magnetization plateau appears, and so on. When $2 S$ spin singlet bonds per three sites are simultaneously broken, there are $2 S$ plateaus occurring. Considering that there is also a saturation plateau, the magnetization per site obeys $m=(S+x) / 3(x=0,1, \cdots, 2 S)$. Therefore, the total number of magnetization plateaus is $2 S+1$, which is well in agreement with the DMRG results[14]. For instance, the trimerized Heisenberg chain with $S=2$, according to our VBS picture, exhibits magnetization plateaus at $m=2 / 3,1,4 / 3,5 / 3$ and 2 , which have been confirmed by the DMRG results[14].

In summary, we have studied the static structure factor $S(q)$ and the plateau states of the F-F-AF and AF-AF-F trimerized Heisenberg chains. It has been found that in plateau states, $S(q)$ with three peaks at $q=0,2 \pi / 3$ and $4 \pi / 3$ does not vary with the magnetic field as well as the exchange couplings $J_{A F} / J_{F}$, and the peak positions are determined by the period of the spin correlation function. From the DMRG data, it is observed that the spin correlation function satisfies a simple relation with the magnetization per site [Eq.(5)]. An approximate trimerized state is presented to describe the physical properties in the plateau states, and the results obtained by using this trial wave function are quite in agreement with the DMRG data. To explain the origin and the total number of the plateaus, a picture in terms of the VBS states is offered. From this picture, the appearance of the magnetization plateaus can be attributed to the breaking of the spin singlet states and the isolated spins. The total number and the positions of the plateaus deduced from our VBS picture are 
exactly the same with the DMRG results. It is expected that the properties of the static structure factors reported in this paper could be useful for future experimental studies on the trimerized Heisenberg spin chain compounds.

\section{Acknowledgments}

We are grateful to X. Chen, Z. C. Wang, Q. B. Yan and Q. R. Zheng for useful discussions. This work is supported in part by the National Science Fund for Distinguished Young Scholars of China (Grant No. 10625419), the National Science Foundation of China (Grant Nos. 90403036 and 20490210), and by the MOST of China (Grant No. 2006CB601102).

[] *Corresponding author. E-mail: gsu@gucas.ac.cn

[1] H. -J. Mikeska and A. K. Kolezhuk, One-Dimensional Magnetism, Lecture Notes in Physics Vol. 645, edited by U. Schollwock, J. Richter, D. J. J. Farnell and R.F. Bishop (SpringerVerlag, Berlin, Heidelberg, 2004).

[2] J. Schulenburg, A. Honecker, J. Schnack, J. Richter, and H. J. Schmidt, Phys. Rev. Lett. 88, 167207 (2002); J. Schnack, H. J. Schmidt, A. Honecker, J. Schulenberg and J. Richter, J. Phys. Condens. Matter 51, 43 (2006).

[3] K. Hida, J. Phys. Soc. Jpn. 63, 2359 (1994).

[4] K. Okamoto, Solid State Commun. 98, 245 (1996).

[5] M. Oshikawa, M. Yamanaka and I. Affleck, Phys. Rev. Lett. 78, 1984 (1997).

[6] A. Kitazawa and K. Okamoto, J. Phys. Condens. Matter 11, 9765 (1999).

[7] A. Honecker, Phys. Rev. B 59, 6790 (1999).

[8] W. Chen, K. Hida and B. C. Sanctuary, J. Phys. Soc. Jpn. 69, 3414 (2000); W. Chen, K. Hida and B. C. Sanctuary, Phys. Rev. B 63, 134427 (2001).

[9] J. Strečka, M. Jaščur, M. Hagiwara, Y. Narumi, K. Kindo, and K. Minami, Phys. Rev. B 72, $024459(2005)$.

[10] H. Kikuchi, Y. Fujii, M. Chiba, S. Mitsudo, T. Idehara, T. Tonegawa, K. Okamoto, T. Sakai, T. Kuwai and H. Ohta, Phys. Rev. Lett. 94, 227201 (2005).

[11] Y. Ajiro, T. Asano, T. Inami, H. Aruga-Katori and T. Goto, J. Phys. Soc. Jpn. 63, 859 (1994). 
[12] M. Ishii, H. Tanaka, M. Hori, H. Uekusa, Y. Ohashi, K. Tatani, Y. Narumi and K. Kindo, J. Phys. Soc. Jpn. 69, 340 (2000).

[13] M. A. M. Abu-Youssef, M. Drillon, A. Escure, M. A. S. Goher, F. A. Mautner and R. Vicente, Inog. Chem. 39, 5022 (2000).

[14] B. Gu, G. Su and S. Gao, J. Phys. Condens. Matter 17, 6081 (2005).

[15] B. Gu, G. Su and S. Gao, Phys. Rev. B 73, 134427 (2006).

[16] B. Gu and G. Su, Phys. Rev. Lett. 97, 089701 (2006).

[17] B. Gu and G. Su, Phys. Rev. B 75, 174437 (2007).

[18] As the lattice of the system can be devided into three sublattices, the static structure factor $S(q)$ could be obtained in terms of a $3 \times 3$ matrix. Our calculations show that, although the sublattice structure factor exhibits different behaviors, the total structure factor $S(q)$ obtained by a $3 \times 3$ matrix gives exactly the same result as that calculated directly by Eq. (2).

[19] S. R. White, Phys. Rev. Lett. 69, 2863 (1992); S. R. White, Phys. Rev. B 48, 10345 (1993).

[20] U. Schollwöck, Rev. Mod. Phys. 77, 259 (2005). 

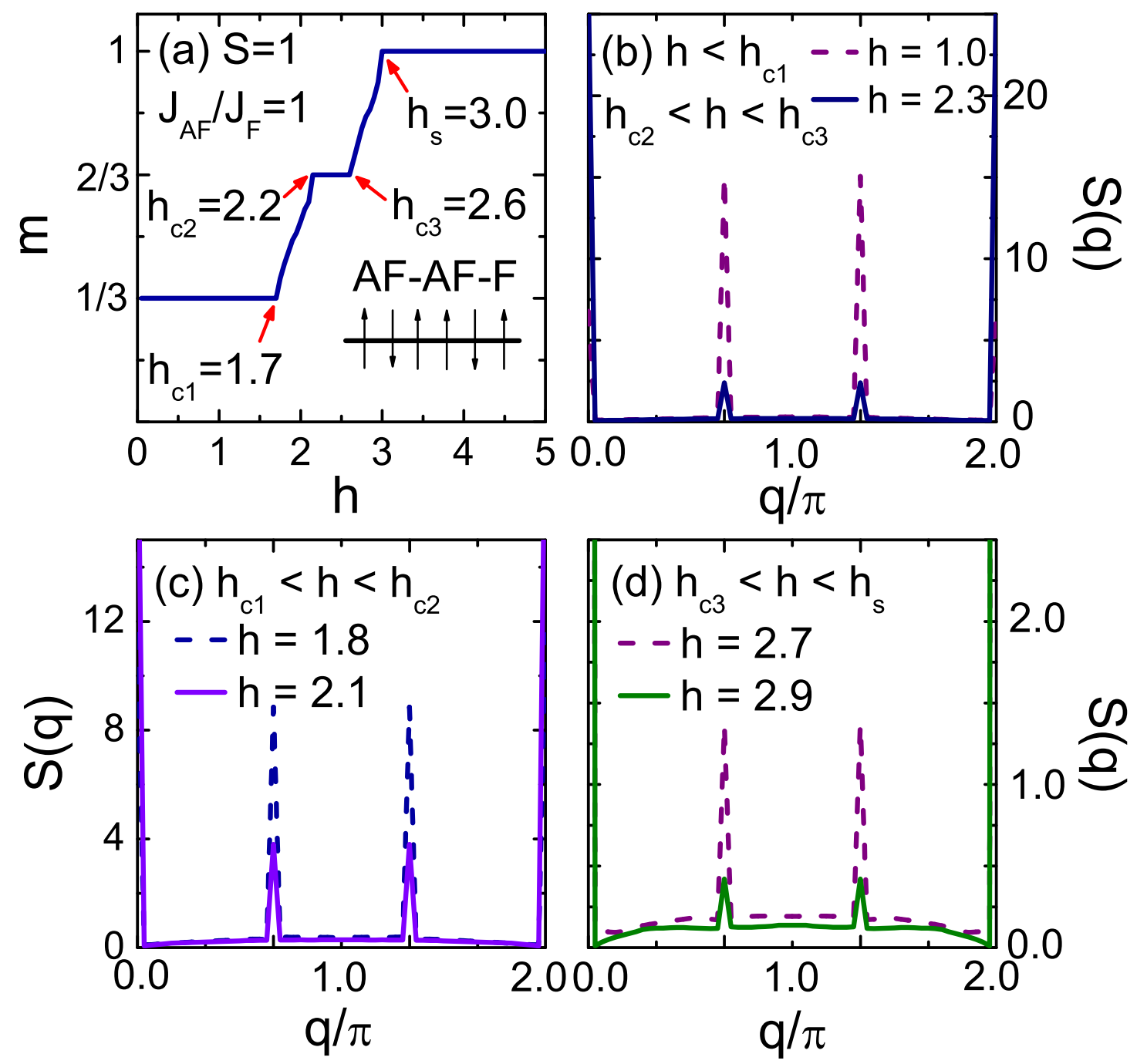

FIG. 2: (Color online) For a spin-1 AF-AF-F trimerized Heisenberg chain with fixed couplings $J_{A F} / J_{F}=1$, (a) the magnetization per site $m$ as a function of the external magnetic field $h$ in the ground states; and the static structure factor $S(q)$ in the ground states with the external field (b) $h<h_{c 1}$ and $h_{c 2}<h<h_{c 3}$, (c) $h_{c 1}<h<h_{c 2}$, (d) $h_{c 3}<h<h_{s}$. 


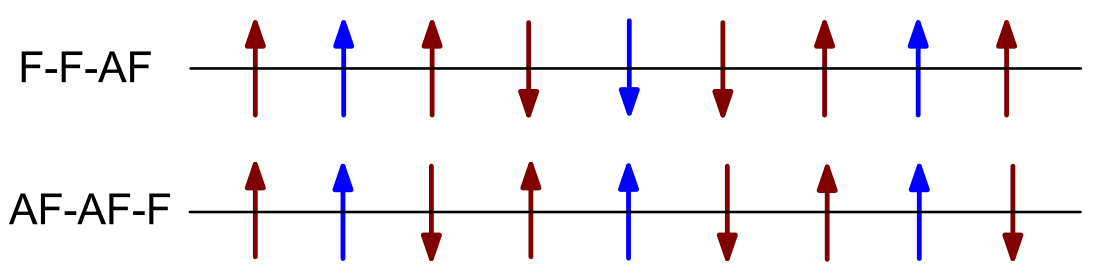

(a)

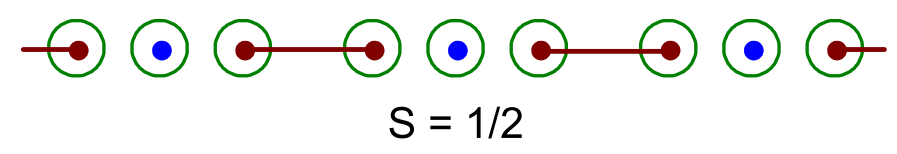

(b)

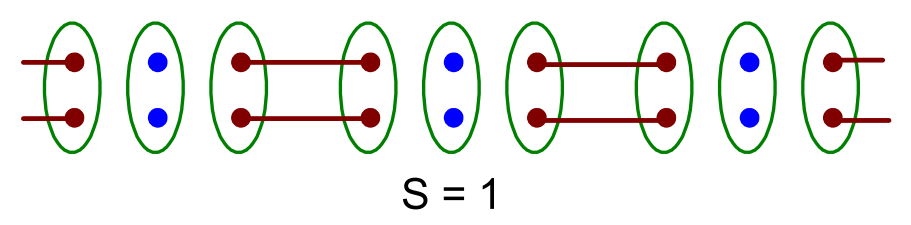

(c)

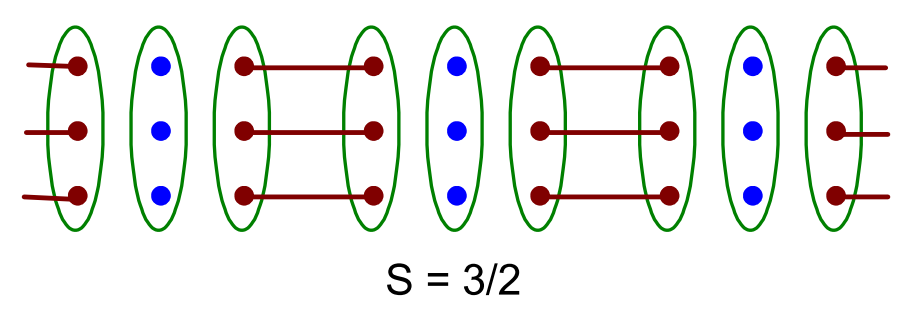

(d)

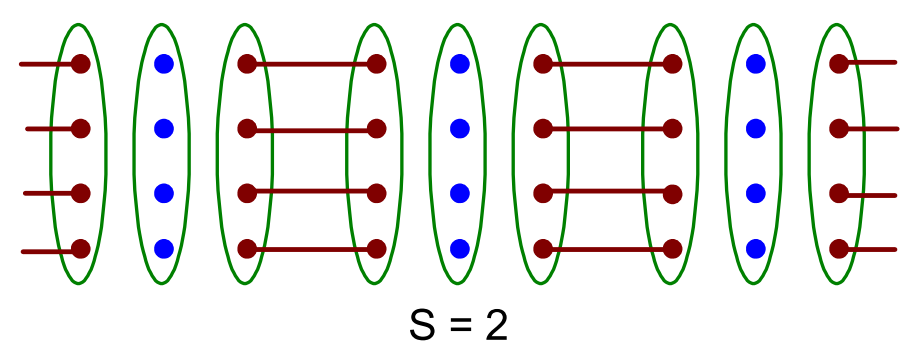

FIG. 3: (Color online) Sketch of the VBS picture of the F-F-AF and AF-AF-F trimerized Heisenberg chains with spin (a) $S=1 / 2$, (b) 1 , (c) $3 / 2$, and (d) 2, respectively. $\uparrow$ denotes spin up, and $\downarrow$ spin down. The bullet $\bullet$ denotes a spin $1 / 2$, and the short line between bullets denotes the spin singlet state. The big open elipse presents spin $S$ per site which could be viewed as a combination of $2 S$ spin $1 / 2$. 